\title{
Evaluating the Headspace Volatolome, Primary Metabolites, and Aroma Characteristics of Koji Fermented with Bacillus amyloliquefaciens and Aspergillus oryzae ${ }^{\mathrm{S}}$
}

\author{
Han Sol Seo ${ }^{1}$, Sunmin Lee ${ }^{1}$, Digar Singh ${ }^{1}$, Min Kyung Park ${ }^{2}$, Young-Suk Kim ${ }^{2}$, Hye Won Shin ${ }^{3}$, Sun A Cho ${ }^{3}$, \\ and Choong Hwan Lee ${ }^{1 *}$ \\ ${ }^{1}$ Department of Bioscience and Biotechnology, Konkuk University, Seoul 05029, Republic of Korea
${ }^{2}$ Department of Food Science and Engineering, Ewha Womans University, Seoul 03760, Republic of Korea
${ }^{3}$ Food Research institute CJ CHEILJEDANG Co., Suwon 16495, Republic of Korea
}

Received: April 16, 2018

Revised: May 23, 2018

Accepted: May 24, 2018

June 8, 2018

${ }^{*}$ Corresponding author

Phone: +82-2-2049-6177;

Fax: +82-2-455-4291;

E-mail: chlee123@kokuk.ac.kr

$\mathrm{S}$ upplementary data for this paper are available on-line only at http://jmb.or.kr.

pISSN 1017-7825, eISSN 1738-8872

Copyright (C) 2018 by

The Korean Society for Microbiology and Biotechnology
First published online

Production of good Koji primarily depends upon the selection of substrate materials and fermentative microflora, which together influence the characteristic flavor and aroma. Herein, we performed comparative metabolomic analyses of volatile organic compounds (VOCs) and primary metabolites for Koji samples fermented individually with Bacillus amyloliquefaciens and Aspergillus oryzae. The VOCs and primary metabolites were analyzed using headspace solid phase microextraction (HS-SPME) followed by gas chromatography time-of-flight mass spectrometry (GC-TOF-MS). In particular, alcohols, ketones, and furans were mainly detected in Bacillus-fermented Koji (Bacillus Koji, BK), potentially due to the increased levels of lipid oxidation. A cheesy and rancid flavor was characteristic of Bacillus Koji, which is attributable to high content of typical 'off-flavor' compounds. Furthermore, the umami taste engendered by 2-methoxyphenol, (E,E)-2,4-decadienal, and glutamic acid was primarily detected in Bacillus Koji. Alternatively, malty flavor compounds (2-methylpropanal, 2-methylbutanal, 3-methylbutanal) and sweet flavor compounds (monosaccharides and maltol) were relatively abundant in Aspergillus-fermented Koji (Aspergillus Koji, AK). Hence, we argue that the VOC profile of Koji is largely determined by the rational choice of inocula, which modifies the primary metabolomes in Koji substrates, potentially shaping its volatolome as well as the aroma characteristics.

Keywords: Koji fermentation, Aspergillus oryzae, Bacillus amyloliquefaciens, mass spectrometry, volatolome, metabolome

\section{Introduction}

Fermentation is a traditional method that makes use of the metabolic activities of microorganisms to improve the content of food and beverages with biomolecules associated with flavor, aroma, as well as organoleptic and nutritional properties [1]. The quintessential Koji gourmet utilizes solid-state fermentation of various substrates materials such as soybeans, wheat, or rice, with a single microbial inocula [2]. During the course of fermentative growth, inoculum such as bacteria or mold species decompose the substrate, resulting in a modified food product [3]. Undoubtedly, Koji represents an important starting ingredient in the manufacturing of a variety of fermented foods and beverages including soybean paste (doenjang, cheonggukjang, miso), soy sauce (kanjang), red pepper paste (gochujang) and rice wine (Makgeolli) [4].

The composition of volatile organic compounds (VOCs) in Koji affects its unique fragrance and aroma and determines its quality characteristics as a raw material component of Koji fermented foods. Moreover, the VOC composition of Koji is considered an important criterion 
toward its consumer acceptability, necessitating the comprehensive analysis of the Koji volatolome. Previously, a variety of VOCs from rice Koji fermented with A. oryzae were reported including alcohols, aldehydes, ketones, and ester compounds [5]. Furthermore, the effects of various fermentative microflora on the flavor compounds in wheat Koji have been evaluated using a permutation of mold inocula viz., Aspergillus, Penicillium, Alternaria, Fusarium, and Cephalosporium species [6]. Similarly, multiple classes of VOC compounds have been reported for Bacillus-Koji doenjang, using HS-SPME-GC-MS methods including esters, alcohols, acids, aldehydes, ketones, sulfur compounds, pyrazines, and volatile phenols [5].

In recent years, an unprecedented improvement in high throughput instrumentation has enabled multiple sampling, enhanced sensitivity, and greater precision. This, coupled with improved data mining algorithms, has brought forth the development of more conventional approaches for VOC analysis. These methods, including gas chromatographyolfaction (GC-O), simultaneous dissolvent extract (SDE), and head-space solid-phase micro-extract gas chromatography mass spectrum (HS-SPME-GC-MS), have been extremely useful in the analysis of VOCs [7]. SPME is a no-solvent method to extract VOCs from solids, liquids, or even gasses. It is considered a highly reproducible method that has been employed in food aroma and perfumery studies $[8,9]$. Importantly, SPME has the ability to extract VOCs from fermented foods, including soy sauce, soy paste, and related beverages $[5,7,10,11]$. Metabolomics has also been used to evaluate the nutritional quality of foods, plants, or fermentation products [12-14]. Recently, we have reported the time-correlated dynamic metabolomes and VOC profiles for the acetous fermentative production stages of vinegar employing the GC-TOF-MS and HS-SPME-GC-MS analyses [15].

A previous study has comprehensively delineated the discriminant metabolomes for rice Koji fermented with two different inocula i.e., A. oryzae and B. amyloliquefaciens, highlighting the differential effects of fermentative microflora on Koji metabolomes [4]. Furthermore, the alterations in respective metabolomes were correlated with corresponding antioxidant levels and enzymatic activities that are considered vital for Koji quality characteristics. However, there have been no previous studies probing the VOC compositions of Koji fermented with different microflora that intertwine the subtle primary metabolomes with VOC profiles and aroma characteristics.

Considering the importance of fermentative microflora for the Koji volatolome, we aim toward comprehensive characterization of the headspace VOCs and primary metabolite profiles for varying Koji (soybean and wheat) samples fermented individually with B. amyloliquefaciens or A. oryzae. Furthermore, the Koji metabolome and volatolome were evaluated to correlate the varying fermentative microflora and aroma characteristics for Koji end products.

\section{Materials and Methods}

\section{Chemicals and Reagents}

HPLC-grade methanol, acetonitrile and water were purchased from Fisher Scientific (USA). L-Borneol and sodium chloride were purchased from Sigma-Aldrich (USA).

\section{Microbial Cultures and Koji (Soybean, Wheat) Fermentation}

As shown in Table 1, the varying fermentative microflora i.e., Bacillus amyloliquefaciens (KCCM 11718P) and Aspergillus oryzae (KCCM 11300P) were procured from the CJ Cheil-Jedang Co., Ltd. (Korea). Two different substrates, soybean (Glycine max) and wheat (Triticum aestivum), were employed for Koji preparation. To make Koji, $1 \mathrm{~kg}$ of substrate was soaked in water for $30 \mathrm{~min}$, and then drained. The soaked substrates were sterilized by autoclaving for $15 \mathrm{~min}$. The steamed substrate $(50 \mathrm{~g})$ was inoculated with A. oryzae and incubated at $37^{\circ} \mathrm{C}$ for 5 days toward the preparation of the Koji pre-inoculum. The pre-inoculum substrate was mixed with the main steamed substrate $(0.2 \%, \mathrm{w} / \mathrm{w})$, and subjected to fermentation $37^{\circ} \mathrm{C}$ for $36 \mathrm{~h}$. The bacterial strain B. amyloliquefaciens was pre-incubated in $200 \mathrm{ml}$ of nutrient broth $(\mathrm{pH} \mathrm{7.0)}$ and incubated at $37^{\circ} \mathrm{C}$ at $200 \mathrm{rpm}$ for $24 \mathrm{~h}$. The cultured broth, serving as the inoculum, was added to the steamed substrate $(2.0 \%, \mathrm{v} / \mathrm{w})$ and fermented at $37^{\circ} \mathrm{C}$ for $36 \mathrm{~h}$. Hence, the four different combinations of Koji including: a. soybean Koji fermented with

Table 1. The information of Koji samples.

\begin{tabular}{llccc}
\hline \multicolumn{1}{c}{ Inoculum } & Substrate & Time $(\mathrm{h})$ & Label & Symbol \\
\hline- & Soybean & 0 & RS & - \\
& Wheat & 0 & RW & $\bigcirc$ \\
B. amyloliquefaciens & Soybean & 12 & BS1 & $\mathbf{\square}$ \\
KCCM 11718P & Soybean & 24 & BS2 & $\nabla$ \\
& Soybean & 36 & BS3 & $\Delta$ \\
& Wheat & 12 & BW1 & $\square$ \\
A. oryzae & Wheat & 24 & BW2 & $\nabla$ \\
KCCM 11300P & Wheat & 36 & BW3 & $\triangle$ \\
& Soybean & 12 & AS1 & $\square$ \\
& Soybean & 24 & AS2 & $\nabla$ \\
& Soybean & 36 & AS3 & $\Delta$ \\
& Wheat & 12 & AW1 & $\square$ \\
& Wheat & 24 & AW2 & $\nabla$ \\
& Wheat & 36 & AW3 & $\triangle$ \\
\hline
\end{tabular}


B. amyloliquefaciens (BS); b. wheat Koji fermented with B. amyloliquefaciens (BW); c. soybean Koji fermented with A. oryzae (AS); and d. wheat Koji fermented with $A$. oryzae (AW) we maintained throughout the experiment (Table 1). The samples were harvested at $12 \mathrm{~h}$ intervals (up to $36 \mathrm{~h}$ ) and were immediately stored at deep freezing conditions $\left(-80^{\circ} \mathrm{C}\right)$ until analyses. The numbers following the letters on the labels are written in order of the different harvest time while fermentation. The harvested Koji samples following 12, 24, and $36 \mathrm{~h}$ of fermentation were indicated as 1, 2, and 3 (Table 1). Three biological replicates were maintained for each sample harvested at aforementioned time periods.

\section{Koji Sample Extraction}

Extraction of headspace volatile organic compounds (VOCs). SPME was used to obtain volatile compounds in Koji samples. Before volatile extraction by SPME, samples were ground using a mortar with liquid nitrogen. Then, each sample $(2.5 \mathrm{~g})$ was mixed with $4 \mathrm{ml}$ of saturated sodium chloride solution to inhibit enzyme activities during the extraction. The sample mixture and $2 \mu \mathrm{l}$ of Lborneol $(200 \mu \mathrm{g} / 1$ in methanol), an internal standard compound, were added into a $20 \mathrm{ml}$ of amber SPME vial with a silicon/teflon septum (Supelco, Bellefonte, PA, USA). The mixtures were maintained at $40^{\circ} \mathrm{C}$ for $30 \mathrm{~min}$ and $75 \mu \mathrm{m}$ carboxen/polydimethylsiloxane/ divinylbenzene (CAR/PDMS/DVB) coated SPME fiber was exposed to the headspace at $40^{\circ} \mathrm{C}$ for $30 \mathrm{~min}$. The desorption procedure was performed at $230^{\circ} \mathrm{C}$ in a GC injector for $5 \mathrm{~min}$.

Extraction for primary metabolites. The freeze-dried Koji samples were ground using a mortar. Extraction of Koji powder $(3 \mathrm{~g})$ was conducted by adding $80 \%$ aqueous methanol $(30 \mathrm{ml})$ and norvaline as an internal standard followed by sonication (10 $\mathrm{min}$ ) and agitation ( $200 \mathrm{rpm}$ for $24 \mathrm{~h}$ ) in a rotary shaker. The sample mixtures were centrifuged (at 2,370 g for $10 \mathrm{~min}$ at $4^{\circ} \mathrm{C}$ ) and the supernatants were filtered using a $0.22 \mu \mathrm{m}$ polytetrafluoroethylene (PTFE) Millex®GP filter (Merck Millipore, Billerica, MA, USA). The sample extracts were completely dried using a speed-vacuum concentrator (Biotron, Korea) and the weighed to estimate the extraction yield. The sample derivatization reaction consisting of two stages, oximation and silylation, was carried out using the methods described previously [4].

\section{Intrumentation}

HS-SPME-GC-MS analysis. GC-MS analysis was conducted using 7890A GC system coupled to 5975C mass detector (Agilent technologies, USA) equipped with a DB-WAX column (30 m length $\times 0.25 \mathrm{~mm}$ internal diameter $\times 0.25 \mu \mathrm{m}$ film thickness, $\mathrm{J} \& \mathrm{~W}$ Scientific, USA). Oven temperature was initially maintained at $40^{\circ} \mathrm{C}$ for $6 \mathrm{~min}$, and then raised to $200^{\circ} \mathrm{C}$ at a rate of $4^{\circ} \mathrm{C} / \mathrm{min}$ before holding at $200^{\circ} \mathrm{C}$ for $5 \mathrm{~min}$. The flow rate of helium was $0.8 \mathrm{ml} / \mathrm{min}$ and mass spectra was obtained with a mass scan range of 35-350 atomic mass units at a rate of $4.5 \mathrm{scans} / \mathrm{sec}$ with $70 \mathrm{eV}$ of ionization energy in EI mode.

GC-TOF-MS analysis. The primary metabolites were analyzed on a GC-TOF-MS using an Agilent 7890A GC system (USA) with an Agilent 7693 auto-sampler and TOF Pegasus HT mass spectrometer (Leco, USA). An RTx-5MS (30 m length $\times 0.25 \mathrm{~mm}$ i.d. $\times 0.25 \mu \mathrm{m}$ film thickness, J \& W Scientific, USA) was used with a carrier gas, helium, at a flow rate of $1.5 \mathrm{ml} / \mathrm{min}$. Transfer line and injector temperatures were set at $230^{\circ} \mathrm{C}$ and $250^{\circ} \mathrm{C}$, respectively. The column temperature was constant at $75^{\circ} \mathrm{C}$ for 2 min, raised to $300^{\circ} \mathrm{C}$ at the rate of $15^{\circ} \mathrm{C} / \mathrm{min}$, and maintained as such for next $3 \mathrm{~min}$. The acquisitions were recorded at the rate of 10 scans/s scanning a mass range of $50-1,000 \mathrm{~m} / \mathrm{z}$, maintaining $70 \mathrm{eV}$ of ionization energy under EI mode. A $1 \mu \mathrm{l}$ of the derivatized sample was injected at a split ratio of 5:1. Three replicates of each sample were tested.

\section{Data Processing and Multivariate Statistical Analysis}

The raw data files of GC-TOF-MS were converted to document format (*.cdf) by LECO Chroma TOF software (Version 4.44). Using the Metalign software package (http://www.metaligh.nl), retention-time correction, peak detection, and accurate masses were processed and exported resulting data to an Excel file (Microsoft, USA). The multivariate statistical analyses were conducted using SIMCA-P+ 12.0 software (Umetrics, Umea, Sweden). The patterns of metabolic variation were visualized using principal component analysis (PCA) and partial leastsquares discriminant analysis (PLS-DA). The detected metabolites tested for significance by a one-way analysis of variance (ANOVA) using STATISTICA software (version 7.0, StatSoft Inc., USA). Primary metabolites were identified by comparing the retention times and mass fragment patterns with the National Institute of Standards and Technology (NIST) database (version 2.0, 2011, FairCom, USA) and those of standard compounds. Volatile compounds were identified by comparing their mass spectral and retention indices (RI) based on Wiley 9th edition mass spectral library (W9N08) and National Institute of Standards Technology version 08 (NIST08) libraries (Agilent Technologies, USA). The RI values were calculated using n-alkane mixture C6 to C22 alkanes as external standards. The relative peak areas of volatile compounds were quantified by comparing their peak areas to that of internal standard compound. The significantly discriminant primary metabolites were selected based on PLS-DA model at VIP $>0.7$ and $p<0.05$. In comparing level of major flavor compounds, significant differences were tested by ANOVA and Duncan's multiple range tests using PASW Statistics 18 (SPAA, Inc., IL). The volatile compounds and primary metabolites levels were visualized using the heat map. Each level of volatile compounds and primary metabolites were converted as the $\log _{10}$ of peak area.

\section{Antioxidant Activity of Koji}

The estimation of antioxidant levels in Koji extracts were made using ABTS [2,2'-azinobis-(3-ethylbenzothiazoline-6-sulfonic acid)] assay adapted from the previously reported protocols [4]. To prepare the assay solution, $7 \mathrm{mM}$ ABTS with $2.45 \mathrm{mM}$ potassium persulfate was incubated under water bath $60^{\circ} \mathrm{C}$ for $20 \mathrm{~min}$ and kept for 
stabilization at room temperature $\left(28^{\circ} \mathrm{C}\right)$ for $12 \mathrm{~h}$. The resulting solution was diluted using distilled water until an absorbance of $0.7 \pm 0.02$ at $750 \mathrm{~nm}$ was achieved under spectrophotometer (Spectronic Genesys 6, Thermo Electron, USA). The Koji sample extract $(20 \mu \mathrm{l})$ was added upon with ABTS $(180 \mu \mathrm{l})$ solution into a 96-well plate, and the reaction mixture was incubated under dark at $28^{\circ} \mathrm{C}$ for $6 \mathrm{~min}$. Finally, the absorbance was recorded at $750 \mathrm{~nm}$. Trolox was used as the reaction standard and the antioxidant activity data were expressed as the trolox equivalents antioxidant capacity (TEAC, mM).

\section{Results}

Time-Correlated Multivariate Analysis of Headspace Volatolome and Primary Metabolite Profiling Datasets for Koji Samples Fermented with Different Inocula

The temporal disparity in the profiles of headspace VOCs and primary metabolites in different Koji types were evaluated using multivariate statistical analyses of the HSSPME-GC-MS and GC-TOF-MS datasets, respectively. As shown in Fig. 1A, the principal component analysis (PCA) score plot based on HS-SPME-GC-MS datasets indicated clustered time-correlated patterns for different Koji types. Notably, the VOCs profiling datasets were clustered each
Koji types based on different inocula (B. amyloliquefaciens and $A$. oryzae) and substrates (soybean and wheat) across PC1 (25\%) and PC2 (21.6\%).

In congruence to the PCA, the corresponding partial least squares discriminant analysis (PLS-DA) score plots displayed similar patterns (Fig. S1A). A total of 141 VOCs were detected in different Koji types (Table S1). These included 7 carboxylic acids, 22 aldehydes, 27 alcohols, 13 esters, 21 ketones, 13 aliphatic hydrocarbons, 7 aromatic hydrocarbons, 5 furans, 5 sulfur-containing compounds, 8 pyrazines, 4 phenols, 2 lactones, and 5 miscellaneous compounds among different Koji types. The VOCs detected from soybean Koji included 7 carboxylic acids, 17 aldehydes, 19 alcohols, 11 esters, 16 ketones, 13 aliphatic hydrocarbons, 7 aromatic hydrocarbons, 3 furans, 5 sulfurcontaining compounds, 5 pyrazines, 4 phenols, 2 lactones, and 3 miscellaneous compounds. Similarly, among the varying VOCs detected from wheat Koji included 4 carboxylic acids, 19 aldehydes, 21 alcohols, 10 esters, 17 ketones, 10 aliphatic hydrocarbons, 5 aromatic hydrocarbons, 4 furans, 5 sulfur-containing compounds, 8 pyrazines, 4 phenols, 1 lactone and 4 miscellaneous compounds. Intriguingly, alcohols were identified as the most abundant
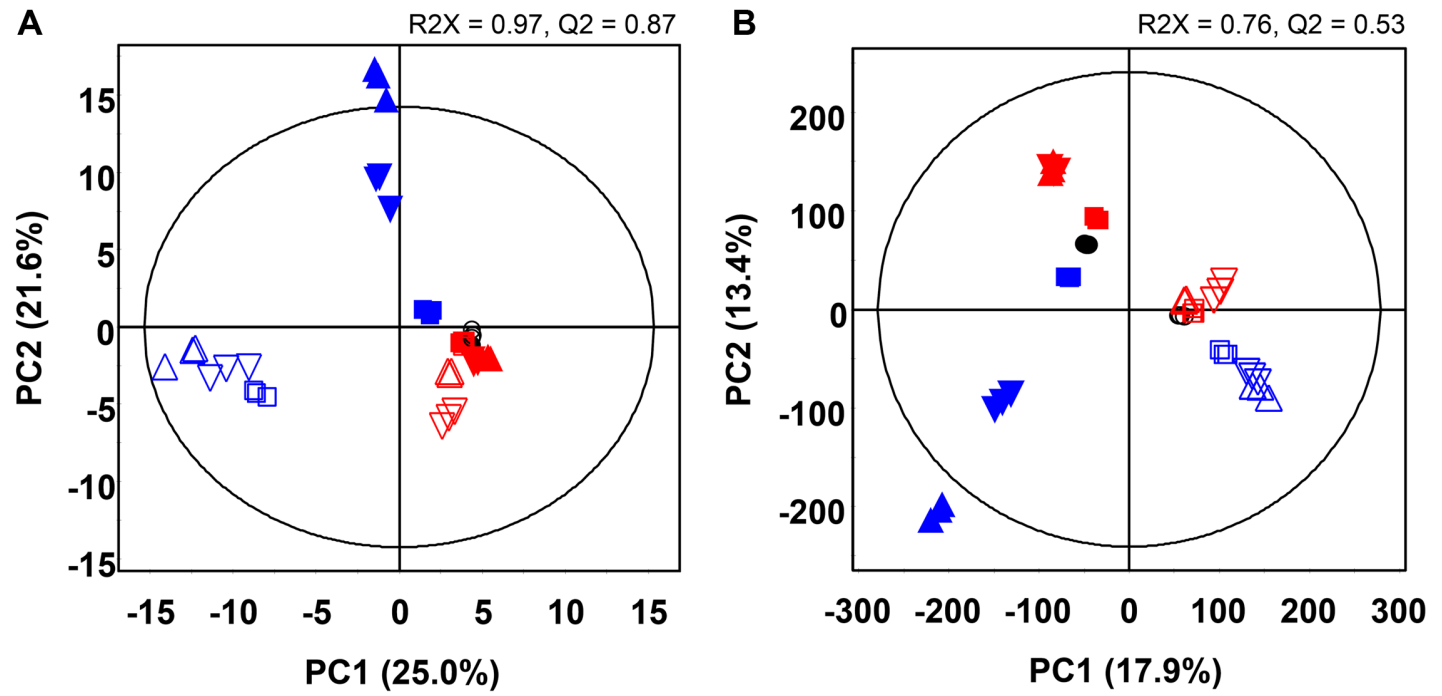

Fig. 1. Principal component analysis (PCA) plots for fermented Koji extracts with different combinations of substrates (soybean and wheat) and fermentative microflora (A. oryzae or B. amyloliquefaciens) during the course of fermentation based on (A) HSSPME-GC-MS datasets for volatolome, and (B) GC-TOF-MS datasets for primary metabolites.

Here, the datasets for raw substrates are indicated - - RS (raw soybean); $\bigcirc$, RW (raw wheat). The datasets for B. amyloliquefaciens fermented soybean samples - $\mathbf{\square}$, BS1 (harvested at 12 h); $\boldsymbol{\nabla}$, BS2 (harvested at 24 h); $\boldsymbol{\Delta}$, BS3 (harvested at 36 h); and wheat samples - $\square$, BW1(harvested at $12 \mathrm{~h}$ ); $\nabla$, BW2 (harvested at $24 \mathrm{~h}$ ); $\triangle$, BW3 (harvested at $36 \mathrm{~h}$ ). Similarly, the datasets for A. oryzae fermented soybean samples - $\square$, AS1 (harvested at $12 \mathrm{~h}$ ); $\boldsymbol{\nabla}, \mathrm{AS} 2$ (harvested at $24 \mathrm{~h}$ ); $\Delta$, AS3 (harvested at $36 \mathrm{~h}$ ); and wheat samples - $\square$, AW1 (harvested at 12 h); $\nabla$, AW2 (harvested at $24 \mathrm{~h}$ ); $\triangle$, AW3 (harvested at $24 \mathrm{~h}$ ). 
VOCs among the different Koji sample extracts, followed by ketones in soybean and aldehydes in wheat Koji samples. The differential variables of each different Koji (BS, BW, AS, AW) were selected based on the variable importance in projection (VIP) values written in the Table S1 and $p$-value $<0.05$ obtained using the PLS-DA (Fig. S1A). The fragrance of each compound in Table S1 was created by reference [3, 5, 16-24].

Considering the variations in primary metabolite profiles based on the GC-TOF-MS datasets (Fig. 1B), the corresponding PCA score plots displayed distinct patterns based on different substrates (soybean and wheat) (PC1: $17.9 \%$ ), and different inocula (B. amyloliquefaciens and A. oryzae) (PC2: 13.4\%). The PLS-DA score plot also indicated a similar metabolome (Fig. S1B). Herein, we identified 13 sugars and sugar alcohols, 12 organic acids, 17 amino acids, 5 fatty acids, 3 nucleosides, and 1 phenolic acid as the primary metabolites among the different Koji sample extracts (Table S2). The differential variables of each different Koji (BS, BW, AS, AW) were selected based on the variable importance in projection (VIP) $>0.7$ values and $p$-value $<0.05$ obtained using the PLS-DA.

\section{Time-Correlated Variations in the Relative Abundance of VOCs and Primary Metabolites among Bacillus- and Aspergillus-Fermented Koji Types}

The marked disparity in the relative levels of metabolites, including VOCs and primary metabolites and the temporal variation among the different Koji types were displayed using a heat map representation of the data (Figs. S2 and S3). Since the commercial end-product Koji samples are generally harvested following $36 \mathrm{~h}$ fermentation, we carried our experiments to $36 \mathrm{~h}$ evaluating the VOC and primary metabolite profiles at every $12 \mathrm{~h}$ intervals. In general, the relative abundance of most VOCs and primary metabolites increased during the course of fermentation.

Notwithstanding different substrate types, the VOCs detected in Bacillus Koji (BS3, BW3) contained higher relative amounts of carboxylic acids (2-methylpropanoic acid, 3-methylbutanoic acid, 3-methylbut-2-enoic acid, 4methylpentanoic acid), alcohols (ethanol, 2-methylbutan-2ol, butan-1-ol, pentan-2-ol, pent-1-en-3-ol, 4-methylpentan2-ol, pentan-1-ol, 5-methylhexan-2-ol, heptan-2-ol, heptan1-ol, 6-methylheptan-2-ol, 5-methylheptan-2-ol, oct-1-en-3ol, 2,5-dimethylhexan-3-ol, 2-ethylhexan-1-ol, butane-2,3diol, octan-1-ol, butane-1,3-diol, nonan-1-ol, (Z)-non-3-en1-ol, phenylmethanol, 2-phenylethanol), esters (methyl acetate, methyl 2-methylpropanoate, ethyl 2-methylpropanoate, methyl 2-methylbutanoate, ethyl 2-methylbutanoate, ethyl 3-methylbutanoate, 3-methylbutyl acetate, methyl 4methylpentanoate, ethyl hexanoate), ketones (propan-2one, butan-2-one, butane-2,3-dione, 3-methylpentan-2-one, 5-methylhexan-2-one, 4-methylpent-3-en-2-one, heptan-2one, 4-methylheptan-2-one, 4,6-dimethylheptan-2-one, 5methylheptan-2-one, 3-hydroxybutan-2-one, octan-2-one, nonan-2-one, decan-2-one, 1-phenylethanone), furans (2methylfuran, 2,3,5-trimethylfuran, 2-butylfuran, 2-pentylfuran), pyrazines (2-methylpyrazine, 2,5-dimethylpyrazine, 2,6dimethylpyrazine, 2,3,5-trimethylpyrazine, 3-ethyl-2,5dimethylpyrazine, 2-ethyl-3,5-dimethylpyrazine, 2,3,5,6tetramethylpyrazine), and phenols (2-methoxyphenol, phenol, 4-ethenyl-2-methoxyphenol, 4-ethenylphenol). However, the relative levels of aldehydes (propanal, 2-methylpropanal, 2-methylbutanal, 3-methylbutanal, 2-methylpentanal, hexanal, (E)-2-methylbut-2-enal, octanal, (E)-hept-2-enal, 2phenylacetaldehyde, 4-methylbenzaldehyde, 2-phenylbut2-enal, (Z)-5-methyl-2-phenylhex-2-enal) were higher in Aspergillus Koji compared to Bacillus Koji (Fig. S2).

In contrast, most of the primary metabolites were detected in higher quantities in Aspergillus Koji (AS3, AW3). These include sugars and sugar alcohols (glycerol, erythritol, fructose, glucose, galactose, sorbitol, myoinositol, glyceryl-glycoside), organic acids (pyruvic acid, succinic acid, glyceric acid, fumaric acid, malic acid, citric acid, gluconic acid), and fatty acids (palmitic acid, linoleic acid, oleic acid). The notable exception were amino acids (alanine, leucine, isoleucine, proline, glycine, serine, threonine, methionine, glutamic acid, phenylalanine, ornithine, lysine, tryptophan), which showed a higher relative abundance in Bacillus Koji compared to Aspergillus Koji (Fig. S3).

\section{Aroma Quality of Volatile Compounds in Koji}

The comparative evaluation of aroma qualities for Bacillus Koji and Aspergillus Koji were presented in Table 2. There are six main flavors associated with Koji including green, cheesy \& rancid, malty, sweet, floral and fruity. In Bacillus Koji, the number of flavor compounds including 3 for 'green' ((E)-oct-2-enal, pent-1-en-3-ol, benzaldehyde), 3 for 'cheesy \& rancid' (2-methylpropanoic acid, 3-methylbutanoic acid, 4-methylpentanoic acid), 4 for 'sweet' (2-butylfuran, 2-pentylfuran, butane-2,3-dione, 4-ethenylphenol) and 9 for 'fruity' (ethyl-2-methylpropanoate, ethyl 2-methylbutanoate, ethyl 3-methylbutanoate, 3-methylbutyl acetate, methyl 4methylpentanoate, ethyl hexanoate, heptan-2-one, octan-2one, nonan-2-one), were detected at relatively higher concentrations compared to Aspergillus Koji. In contrast, the number of malty flavor compounds (2-methylpropanal, 2- 
Table 2. The relative contents of volatile compound-related flavor in soybean and wheat Koji after $36 \mathrm{~h}$ fermentation either with A. oryzae or B. amyloliquefaciens.

\begin{tabular}{|c|c|c|c|c|}
\hline \multicolumn{5}{|l|}{ Green flavor } \\
\hline \multirow{2}{*}{ Compounds } & \multicolumn{2}{|c|}{ B. amyloliquefacience } & \multicolumn{2}{|c|}{ A. oryzae } \\
\hline & Soybean Koji & Wheat Koji & Soybean Koji & Wheat Koji \\
\hline (E)-Oct-2-enal & N.D. & $1.31 \pm 0.05$ & N.D. & $1.26 \pm 0.05$ \\
\hline Pent-1-en-3-ol & $1.08 \pm 0.04$ & $1.11 \pm 0.03$ & N.D. & N.D. \\
\hline Benzaldehyde & $2.50 \pm 0.10$ & $2.17 \pm 0.03$ & $2.24 \pm 0.03$ & $2.16 \pm 0.04$ \\
\hline Acetaldehyde & N.D. & $1.78 \pm 0.07$ & $2.04 \pm 0.12$ & $1.70 \pm 0.05$ \\
\hline \multicolumn{5}{|l|}{ Cheesy, Rancid flavor } \\
\hline \multirow{2}{*}{ Compounds } & \multicolumn{2}{|c|}{ B. amyloliquefacience } & \multicolumn{2}{|c|}{ A. oryzae } \\
\hline & Soybean Koji & Wheat Koji & Soybean Koji & Wheat Koji \\
\hline 2-Methylpropanoic acid & $2.81 \pm 0.15$ & $2.36 \pm 0.01$ & $1.24 \pm 0.04$ & $1.29 \pm 0.02$ \\
\hline 3-Methylbutanoic acid & $2.76 \pm 0.11$ & $3.22 \pm 0.01$ & $1.79 \pm 0.08$ & $2.31 \pm 0.01$ \\
\hline 4-Methylpentanoic acid & $1.82 \pm 0.12$ & N.D. & N.D. & N.D. \\
\hline Acetic acid & N.D. & $2.27 \pm 0.11$ & $2.08 \pm 0.06$ & $1.35 \pm 0.05$ \\
\hline Butanoic acid & $0.93 \pm 0.10$ & $1.56 \pm 0.12$ & N.D. & $1.57 \pm 0.10$ \\
\hline 3-Methylbutan-1-ol & $2.25 \pm 0.02$ & $2.10 \pm 0.05$ & $1.50 \pm 0.08$ & $2.37 \pm 0.00$ \\
\hline \multicolumn{5}{|l|}{ Malty flavor } \\
\hline \multirow{2}{*}{ Compounds } & \multicolumn{2}{|c|}{ B. amyloliquefacience } & \multicolumn{2}{|c|}{ A. oryzae } \\
\hline & Soybean Koji & Wheat Koji & Soybean Koji & Wheat Koji \\
\hline 2-Methylpropanal & N.D. & $0.89 \pm 0.10$ & $2.30 \pm 0.03$ & $1.86 \pm 0.02$ \\
\hline 2-Methylbutanal & $1.14 \pm 0.07$ & $1.72 \pm 0.14$ & $3.03 \pm 0.07$ & $2.13 \pm 0.03$ \\
\hline 3-Methylbutanal & $1.12 \pm 0.05$ & $2.02 \pm 0.15$ & $3.55 \pm 0.06$ & $3.10 \pm 0.01$ \\
\hline \multicolumn{5}{|l|}{ Sweet flavor } \\
\hline \multirow{2}{*}{ Compounds } & \multicolumn{2}{|c|}{ B. amyloliquefacience } & \multicolumn{2}{|c|}{ A. oryzae } \\
\hline & Soybean Koji & Wheat Koji & Soybean Koji & Wheat Koji \\
\hline 2-Butylfuran & N.D. & $0.85 \pm 0.05$ & N.D. & N.D. \\
\hline 2-Pentylfuran & $2.08 \pm 0.19$ & $2.40 \pm 0.03$ & $2.06 \pm 0.12$ & $0.79 \pm 0.08$ \\
\hline Butane-2,3-dione & $2.05 \pm 0.06$ & $2.57 \pm 0.04$ & $1.77 \pm 0.11$ & $1.73 \pm 0.03$ \\
\hline 4-Ethenylphenol & $2.04 \pm 0.04$ & $0.64 \pm 0.08$ & N.D. & N.D. \\
\hline Hexanal & $2.07 \pm 0.14$ & $2.42 \pm 0.07$ & $2.33 \pm 0.13$ & $2.82 \pm 0.04$ \\
\hline 2-Ethylfuran & $1.40 \pm 0.04$ & N.D. & $1.46 \pm 0.07$ & N.D. \\
\hline Maltol & $1.41 \pm 0.07$ & N.D. & $1.97 \pm 0.05$ & N.D. \\
\hline 3-Methylbut-2-enal & N.D. & N.D. & $1.05 \pm 0.07$ & $1.00 \pm 0.06$ \\
\hline \multicolumn{5}{|l|}{ Floral flavor } \\
\hline \multirow{2}{*}{ Compounds } & \multicolumn{2}{|c|}{ B. amyloliquefacience } & \multicolumn{2}{|c|}{ A. oryzae } \\
\hline & Soybean Koji & Wheat Koji & Soybean Koji & Wheat Koji \\
\hline 2-Phenylacetaldehyde & $1.09 \pm 0.01$ & $1.30 \pm 0.06$ & $1.64 \pm 0.08$ & $1.36 \pm 0.01$ \\
\hline 2-Phenylbut-2-enal & N.D. & N.D. & $2.00 \pm 0.06$ & $0.88 \pm 0.01$ \\
\hline 2-Phenylethanol & $0.73 \pm 0.06$ & $1.33 \pm 0.14$ & N.D. & $0.36 \pm 0.09$ \\
\hline Phenol & $1.42 \pm 0.02$ & $1.51 \pm 0.07$ & N.D. & $0.60 \pm 0.04$ \\
\hline
\end{tabular}


Table 2. Continued.

\begin{tabular}{|c|c|c|c|c|}
\hline \multicolumn{5}{|l|}{ Fruity flavor } \\
\hline \multirow{2}{*}{ Compounds } & \multicolumn{2}{|c|}{ B. amyloliquefacience } & \multicolumn{2}{|c|}{ A. oryzae } \\
\hline & Soybean Koji & Wheat Koji & Soybean Koji & Wheat Koji \\
\hline Ethyl 2-methylpropanoate & $1.37 \pm 0.05$ & $0.85 \pm 0.19$ & $0.57 \pm 0.02$ & N.D. \\
\hline Ethyl 2-methylbutanoate & $1.67 \pm 0.07$ & N.D. & N.D. & N.D. \\
\hline Ethyl 3-methylbutanoate & N.D. & N.D. & N.D. & $1.39 \pm 0.11$ \\
\hline 3-Methylbutyl acetate & N.D. & $0.95 \pm 0.05$ & N.D. & $0.73 \pm 0.07$ \\
\hline Methyl 4-methylpentanoate & $1.10 \pm 0.04$ & N.D. & N.D. & N.D. \\
\hline Ethyl hexanoate & $2.15 \pm 0.04$ & $1.80 \pm 0.11$ & N.D. & $0.59 \pm 0.07$ \\
\hline Heptan-2-one & $2.43 \pm 0.07$ & $2.34 \pm 0.15$ & $0.86 \pm 0.10$ & $0.88 \pm 0.08$ \\
\hline Octan-2-one & $1.13 \pm 0.09$ & $2.47 \pm 0.10$ & $0.69 \pm 0.13$ & N.D. \\
\hline Nonan-2-one & $2.04 \pm 0.01$ & $1.62 \pm 0.04$ & N.D. & N.D. \\
\hline (E)-Hept-2-enal & N.D. & N.D. & N.D. & $1.75 \pm 0.08$ \\
\hline 2-Phenylethyl acetate & N.D. & N.D. & $0.35 \pm 0.06$ & N.D. \\
\hline Ethyl acetate & $1.60 \pm 0.11$ & $2.96 \pm 0.04$ & $2.49 \pm 0.13$ & $2.83 \pm 0.13$ \\
\hline
\end{tabular}

${ }^{\mathrm{a}}$ The value means $\log$ (peak area) \pm STD.

${ }^{\mathrm{b}}$ N.D. means not detected.

methylbutanal, 3-methylbutanal) were relatively abundant in Aspergillus Koji compared to Bacillus Koji. However, the number of floral flavor compounds in both the Bacillus and Aspergillus fermented Koji types were commensurate to their respective headspace abundance irrespective of the varying substrates.

\section{Discussion}

The present study is designed to explore the intricate relationship between subtle primary metabolites and the flavor-imparting VOCs in different types of Koji fermented with different inocula. The corresponding metabolic pathways indicated common trends, regardless of the varying substrates, between Koji sample extracts fermented either with B. amyloliquefaciens or A. oryzae (Fig. 2).

The biosynthetic pathway map indicates the metabolites and their relative occurrence in each of the Koji types (Bacillus Koji than Aspergillus Koji) and is based on the KEGG database and previously published literature $[5,10$, $11,16,17,25]$. Considering some of the selected VOC and primary metabolite levels, we will discuss the potential biosynthetic mechanisms substantiating this observed metabolic disparity between the varying Koji types with two different inocula. Reportedly, 3-hydroxybutan-2-one (acetoin) synthesis in B. subtilis involves two enzymes, which catalyze the conversion of pyruvate to acetolactate (acetolactate synthase) and its subsequent conversion to acetoin (acetolactate decarboxylase) [26]. Hence, we suggest that the coherent activities of these enzymes may have affected the higher relative abundance of 3hydroxybutan-2-one in Bacillus Koji, while the higher pyruvate levels detected in Aspergillus Koji may be attributed to the defective function of these pathways (Fig. 2). The higher phenylacetaldehyde levels in Koji samples fermented with $A$. oryzae can be correlated to the higher expression of enzymes such as aromatic amino acid decarboxylase and monoamine oxidase [27].

The analysis of flavor (green, cheesy, sweet, rancid, and fruity)-related VOCs using HS-SPME-GC-MS indicated that most of the acids, alcohols, esters, ketones, furans, pyrazines, and phenols were generally more abundant in Bacillus fermented Koji, with the exception of some sweet flavor compounds, including maltol, hexanal, and 2ethylfuran. The engendering sweet flavor of VOCs, attributed to hexanal, 2-ethylfuran, 3-hydroxy-2-methyl4H-pyran-4-one (maltol), 2-butylfuran, 2-pentylfuran, butane-2,3-dione, 4-ethenylphenol, and 3-methylbut-2enal, have been previously characterized [17-19]. Since, maltol is produced through the sugar Maillard reaction [17], the higher relative levels of monosaccharide sugars in Aspergillus Koji produced by this reaction may have added to its sweet flavor (Fig. S3). Notably, VOCs and metabolites associated with sweet flavor, such as maltol, 2-ethylfuran, 


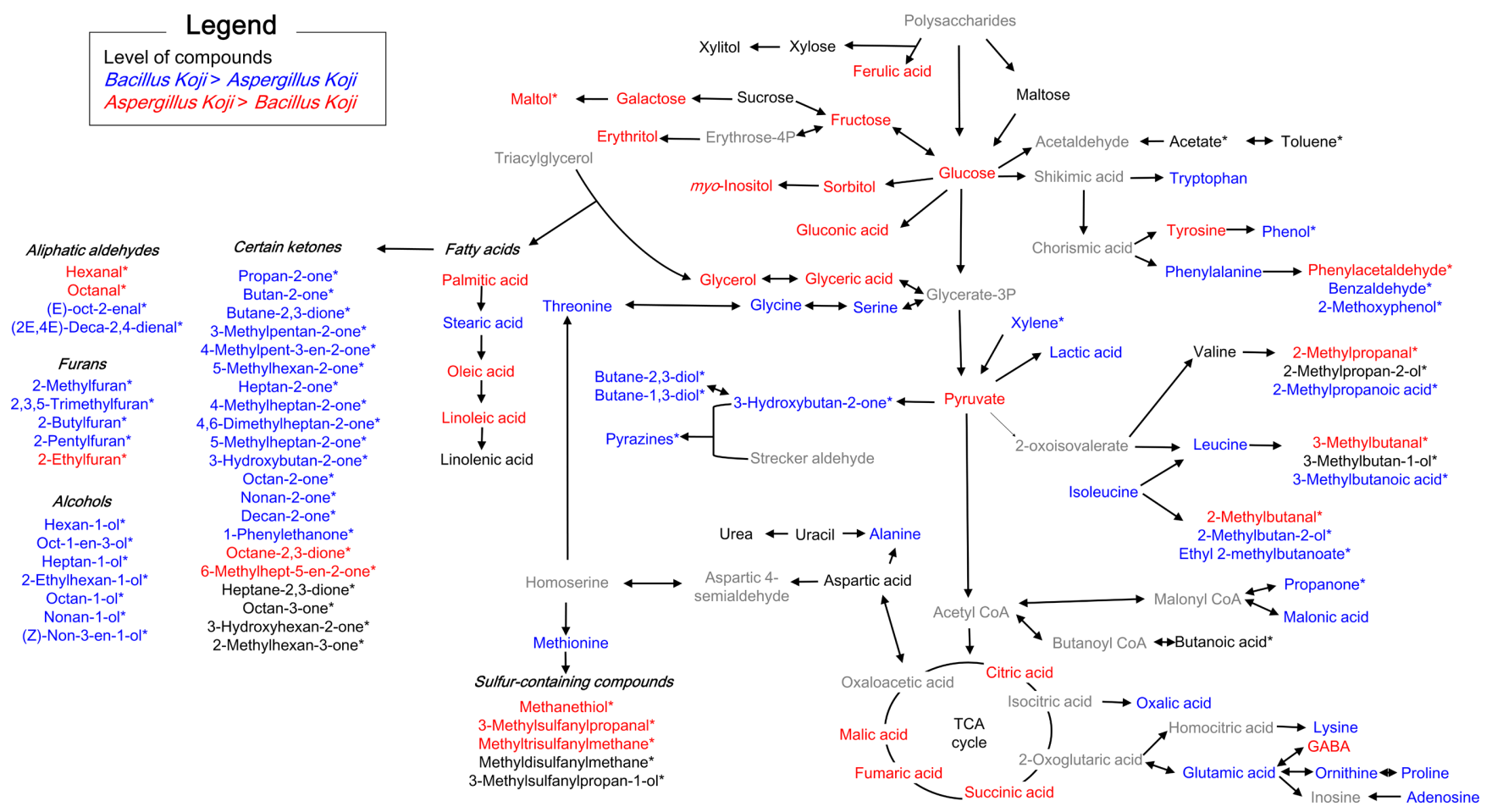

Fig. 2. Scheme of the primary metabolic pathway and volatile compound formation in soybean and wheat Koji fermented after 36 $\mathrm{h}$ with either with A. oryzae or B. amyloliquefaciens.

The pathway was adopted from the KEGG database (KEGG, http://www.genome.jp/kegg) and references. ${ }^{*}$ Volatile compounds; Color of compound name: Blue, higher levels in Bacillus Koji than Aspergillus Koji; Red, higher levels in Aspergillus Koji than Bacillus Koji; Black, Detected compounds regardless of inocula; Gray, Non-detected compounds in Koji.

hexanal, and monosaccharides, were detected in higher levels in Aspergillus Koji (Table 2). Maltol has been previously detected as a major VOC using the solvent-assisted flavor evaporation (SAFE) method in the headspace of fermented soybean paste doenjang [11].

The 'cheesy \& rancid' flavor compounds including 2-methyl butanoic acid and 3-methyl butanoic acid were regarded as off-flavor VOCs in doenjang fermented with B. amyloliquefaciens [5]. Furthermore, VOCs including 3methylbutanal, 2-methylbutanal, 2-methoxyphenol, and (E,E)-2,4-decadienal typically generate the umami aftertaste flavor in miso [3]. In addition, glutamic acid and aspartic acid are amino acids (primary metabolites) known for contributing toward umami taste in fermented foods. Among these compounds, 2-methoxyphenol, (E,E)-2,4decadienal, and glutamic acid were mainly detected from Bacillus Koji regardless of substrates, and can, therefore, be correlated to the fermentative growth of B. amyloliquefaciens (Figs. 2 and S2).

Aliphatic aldehydes, furans, alcohols, and certain ketones are usually formed through lipid oxidation $[5,10]$. In the present study, we determined a higher abundance of these VOCs from Bacillus Koji. Furans are formed from the autooxidation products of linolenic acid and sugar dehydration during the Maillard reaction [5, 17]. However, the antioxidant inhibits the oxidation of unsaturated fatty acids including linolenic acid and linoleic acid, and it can effectively limit the furan production [28]. Based on the results of the ABTS assay, Aspergillus Koji extracts displayed higher antioxidant activity than Bacillus Koji (Fig. S4). Hence, it can be assumed that the lipid oxidation occurred at higher levels in Bacillus Koji than in Aspergillus Koji, and thus, VOCs, including aliphatic aldehydes, furans, alcohols, and certain ketones, were more abundant in Bacillus Koji. Sulfur-containing compounds are mainly produced by the degradation of methionine during the Maillard reaction [11, 16]. Therefore, the higher abundance of Sulfur-containing compounds in Aspergillus Koji may be attributed to the higher free sugar contents, which promote the Maillard reaction. Although, the levels of methionine were relatively higher in Bacillus Koji, the lower occurrence of Maillard reaction likely resulted in the lower abundance 
of organo-sulfur compounds. Hence, the marked disparity in the levels of VOCs and primary metabolites in different Koji types fermented with varying inocula greatly affects the end-product flavor, which is potentially vital for its consumer acceptability and commercial value.

Recapitulating the main findings, we examined 141 headspace VOCs and 51 primary metabolites in different Koji types. We noted higher abundance of carboxylic acids, alcohols, esters, ketones, furans, pyrazines, phenols, and amino acids in Bacillus Koji, regardless of the substrate (soybean or wheat). In contrast, aldehydes, sugars and sugar alcohols, organic acids, and fatty acids were at higher concentrations in Aspergillus Koji. A majority of the flavorrelated VOCs were detected in Bacillus Koji, especially green, cheesy \& rancid, sweet, and fruity flavors. Moreover, the aftertaste flavor compounds including glutamic acid, (E,E)-2,4-decadienal, and 2-methoxyphenol associated with umami taste were detected in higher amounts in Bacillus Koji. Alternatively, Aspergillus Koji displayed higher abundance of malty as well as selected sweet flavor VOCs (maltol, hexanal, 2-ethylfuran) and metabolites (monosaccharides). Bacillus Koji showed more compounds associated with sweet flavor than Aspergillus Koji. However, the primary metabolites, especially the monosaccharides engendering sweet taste, were relatively higher in Aspergillus Koji. Additionally, only two VOCs were detected in Aspergillus Koji and Bacillus Koji related to the umami taste. However, the analysis of primary metabolites also revealed that the number of umami related compounds in Bacillus Koji were higher than those in Aspergillus Koji. Further, these results highlight the need for an integrated approach correlating the VOCs and metabolites with different fermentative inocula toward optimal Koji making. Hence, the present study holds the potential toward engineering the flavor volatolome in fermented foods through a rational choice of fermentative microflora, thus maneuvering the consumer acceptance of fermented end-products.

\section{Acknowledgments}

This research was supported by Traditional Culture Convergence Research Program through the National Research Foundation of Korea (NRF) funded by the Ministry of Science, ICT \& Future Planning (NRF-2017M3C1B5019303).

\section{Conflict of Interest}

The authors have no financial conflicts of interest to declare.

\section{References}

1. Lee DE, Lee S, Singh D, Jang ES, Shin HW, Moon BS, et al. 2017. Time-resolved comparative metabolomes for Koji fermentation with brown-, white-, and giant embryo-rice. Food Chem. 231: 258-266.

2. Okutsu K, Yoshizaki Y, Ikeda N, Kusano T, Hashimoto F, Takamine K. 2015. Antioxidants in heat-processed koji and the production mechanisms. Food Chem. 187: 364-369.

3. Inoue Y, Kato S, Saikusa M, Suzuki C, Otsubo Y, Tanaka Y, et al. 2016. Analysis of the cooked aroma and odorants that contribute to umami aftertaste of soy miso (Japanese soybean paste). Food Chem. 213: 521-528.

4. Lee DE, Lee S, Jang ES, Shin HW, Moon BS, Lee CH. 2016. Metabolomic profiles of Aspergillus oryzae and Bacillus amyloliquefaciens during rice koji fermentation. Molecules 21: 773.

5. Hong Y, Jung HJ, Kim HY. 2012. Aroma characteristics of fermented Korean soybean paste (Doenjang) produced by Bacillus amyloliquefaciens. Food Sci. Biotechnol. 21: 1163-1172.

6. Kaminski E, Stawicki S, Wasowicz E. 1974. Volatile flavor compounds produced by molds of Aspergillus, Penicillium, and Fungi imperfecti. Appl. Microbiol. 27: 1001-1004.

7. Yanfang Z, Wenyi T. 2009. Flavor and taste compounds analysis in Chinese solid fermented soy sauce. Afr. J. Biotechnol. 8: 673-681.

8. Jeleń HH, Wlazły K, Wạsowicz E, Kamiński E. 1998. Solidphase microextraction for the analysis of some alcohols and esters in beer: comparison with static headspace method. J. Agric. Food Chem. 46: 1469-1473.

9. Setkova L, Risticevic S, Pawliszyn J. 2007. Rapid headspace solid-phase microextraction-gas chromatographic-time-offlight mass spectrometric method for qualitative profiling of ice wine volatile fraction: II: classification of Canadian and Czech ice wines using statistical evaluation of the data. J. Chromatogr. A 1147: 224-240.

10. Feng Y, Cui C, Zhao H, Gao X, Zhao M, Sun W. 2013. Effect of koji fermentation on generation of volatile compounds in soy sauce production. Int. J. Food Sci. Technol. 48: 609-619.

11. Jo YJ, Cho IH, Song CK, Shin HW, Kim YS. 2011. Comparison of fermented soybean paste (Doenjang) prepared by different methods based on profiling of volatile compounds. J. Food Sci. 76: 368-379.

12. Lee GM, Suh DH, Jung ES, Lee CH. 2016. Metabolomics provides quality characterization of commercial gochujang (fermented pepper paste). Molecules 21: 921.

13. Lee MY, Singh D, Kim SH, Lee SJ, Lee CH. 2016. Ultrahigh pressure processing produces alterations in the metabolite profiles of Panax ginseng. Molecules 21: 816.

14. Lee S, Oh DG, Lee S, Kim GR, Lee JS, Son YK, et al. 2015. Chemotaxonomic metabolite profiling of 62 indigenous plant species and its correlation with bioactivities. Molecules 20: 19719-19734. 
15. Lee MY, Kim HY, Singh D, Yeo SH, Baek SY, Park YK, et al. 2017. Construing temporal metabolomes for acetous fermentative production of Rubus coreanus vinegar and its in vivo nutraceutical effects. J. Funct. Food. 34: 311-318.

16. Chung HY, Fung PK, Kim JS. 2005. Aroma impact components in commercial plain sufu. J. Agric. Food Chem. 53: 1684-1691.

17. Fors S. 1983. Sensory properties of volatile Maillard reaction products and related compounds: A literature review. ACS Symp Ser Am Chem Soc. 215: 185-286.

18. Feng Y, Su G, Zhao H, Cai Y, Cui C, Sun-Waterhouse D, et al. 2015. Characterisation of aroma profiles of commercial soy sauce by odour activity value and omission test. Food Chem. 167: 220-228.

19. Peng X, Li X, Shi X, Guo S. 2014. Evaluation of the aroma quality of Chinese traditional soy paste during storage based on principal component analysis. Food Chem. 151: 532-538.

20. Kang KM, Baek HH. 2014. Aroma quality assessment of Korean fermented red pepper paste (gochujang) by aroma extract dilution analysis and headspace solid-phase microextractiongas chromatography-olfactometry. Food Chem. 145: 488-495.

21. Dongmo SN, Sacher B, Kollmannsberger H, Becker T. 2017. Key volatile aroma compounds of lactic acid fermented malt based beverages-impact of lactic acid bacteria strains. Food
Chem. 229: 565-573.

22. Cheng H. 2010. Volatile flavor compounds in yogurt: a review. Crit. Rev. Food Sci. Nutr. 50: 938-950.

23. Molimard P, Spinnler HE. 1996. Compounds involved in the flavor of surface mold-ripened cheeses: origins and properties. J. Dairy Sci. 79: 169-184.

24. Morales MT, Rios JJ, Aparicio R. 1997. Changes in the volatile composition of virgin olive oil during oxidation: flavors and off-flavors. J. Agric. Food Chem. 45: 2666-2673.

25. Cho IH, Kim SY, Choi HK, Kim YS. 2006. Characterization of aroma-active compounds in raw and cooked pinemushrooms (Tricholoma matsutake Sing.). J. Agric. Food Chem. 54: 6332-6335.

26. Ryu CM, Farag MA, Hu CH, Reddy MS, Wei HX, Paré PW, et al. 2003. Bacterial volatiles promote growth in Arabidopsis. Proc. Natl. Acad. Sci. USA 100: 4927-4932.

27. Masuo S, Osada L, Zhou S, Fujita T, Takaya N. 2015. Aspergillus oryzae pathways that convert phenylalanine into the flavor volatile 2-phenylethanol. Fungal Genet. Biol. 77: 22-30.

28. Teng D, Gao M, Yang Y, Liu B, Tian Z, Wang J. 2012. Biomodification of soybean meal with Bacillus subtilis or Aspergillus oryzae. Biocatal. Agric. Biotechnol. 1: 32-38. 